\title{
Article
}

\section{The Metabolic Stability of Antimicrobial Peptides IK8 in Plasma and Liver S9}

\author{
Yingying Mao ${ }^{1,+}$, Shaojun Zhou ${ }^{1,+}$, Mingcheng Xu ${ }^{1}$, Su Zeng ${ }^{1} \mathbb{D}$, Weimin Fan ${ }^{2}$, Lushan Yu ${ }^{1, *}$ \\ and Nengming Lin $3, *$
}

1 Institute of Drug Metabolism and Pharmaceutical Analysis, College of Pharmaceutical Sciences, Zhejiang University, Hangzhou 310058, China; adeline219@163.com (Y.M.); zhoushaojun2000@163.com (S.Z.); xumingcheng1989@163.com (M.X.); zengsu@zju.edu.cn (S.Z.)

2 Program of Innovative Cancer Therapeutics, Division of Hepatobiliary and Pancreatic Surgery, Department of Surgery, College of Medicine, Zhejiang University, Hangzhou 310003, China; fanw@zju.edu.cn

3 Key Laboratory of Clinical Cancer Pharmacology and Toxicology Research of Zhejiang Province, Department of Clinical Pharmacy, Zhejiang University School of Medicine, Hangzhou 310006, China

* Correspondence: yuls@zju.edu.cn (L.Y.); lnm1013@zju.edu.cn (N.L.); Tel.: +86-571-88208407 (L.Y.); +86-571-56007905 (N.L.)

+ These authors contributed equally to this work.

Citation: Mao, Y.; Zhou, S.; Xu, M.; Zeng, S.; Fan, W.; Yu, L.; Lin, N. The Metabolic Stability of Antimicrobial Peptides IK8 in Plasma and Liver S9. Appl. Sci. 2021, 11, 11661. https:// doi.org/10.3390/app112411661

Academic Editor: Chiara Cavaliere

Received: 20 October 2021

Accepted: 3 December 2021

Published: 8 December 2021

Publisher's Note: MDPI stays neutral with regard to jurisdictional claims in published maps and institutional affiliations.

Copyright: (c) 2021 by the authors. Licensee MDPI, Basel, Switzerland. This article is an open access article distributed under the terms and conditions of the Creative Commons Attribution (CC BY) license (https:// creativecommons.org/licenses/by/ $4.0 /)$.

\begin{abstract}
In the face of mounting global antibiotic resistance, which has become a critical clinical problem, antimicrobial peptides (AMPs) have received considerable interest as new therapeutics with the efficacy for the treatment of multidrug-resistant (MDR) infections due to their novel mechanism. However, certain inherent shortcomings such as instability seriously limit their systemic applications in the clinic. In this study, we intend to clarify the connection between three configurations of IK8 and their stability in plasma and liver S9 of various species by confirming the metabolites. The structural information of these metabolites was scanned and identified using HPLC and Q-TOF, respectively. The results found that IK8-D exhibits superior stability, compared with IK8-2D and IK8-L in plasma and liver S9 incubation, which indicated D- type amino acids could significantly increase the stability of antimicrobial peptides.
\end{abstract}

Keywords: antimicrobial peptide; IK8; metabolite; Q-TOF

\section{Introduction}

A variety of antimicrobial agents are available today, and they are broadly applied to treat a number of human infections. Specifically, antibiotics are powerful drugs used for the treatment of pathogenic bacteria [1]. However, due to their indiscriminate and prolonged use, at present, multidrug-resistant (MDR) and even pandrug-resistant (PDR) bacteria have spread widely around the world and are currently responsible for increased morbidity and mortality rates, as well as the significant cost to society [2]. With the low frequency of discoveries of new classes of antibiotics and the rapid emergence of resistance to novel antibiotics [3], the design and identification of alternative classes of antimicrobial agents with new modes of action that can effectively overcome MDR are more pressing than ever.

Data from both the laboratory and the clinic in recent years have indicated that antimicrobial peptides (AMPs) exert activity against a broad spectrum of bacteria and fungi, which has captured great attention from academic and clinical sectors. There are several reports that can be cited to illuminate it. First, as an AMP originated from Coprinopsis cinerea (Mushroom), copsin is bactericidal for a diversity of Gram-positive bacteria via disturbing the biosynthesis of the cell wall, including human pathogens such as Listeria monocytogenes [4]. Moreover, cecropins derived from the hemolymph of Hyalophora cecropia, have a potent antibacterial effect on Beauveria bassiana as well as Aedes aegypti [5]. Currently, Wang et al. [6] have discovered a new short non-ribosomal 
AMP, albopeptide, in the culture broth of Streptomyces albofaciens, which displays a narrow-spectrum activity against vancomycin-resistant Enterococcus faecium.

AMPs are bioactive small proteins, as the first-line defense against microbial attacks in Eukaryotes or a competitive strategy in Prokaryotes, generally containing both cationic and hydrophobic amino acids [7]. AMPs exhibit their antimicrobial effects mainly through two different mechanisms. The membrane-targeting AMPs destroy the structural integrity of the cell membrane, while the AMPs that use non-membrane targeting mechanisms principally inhibit the synthesis of nucleic acids, essential enzymes, and other functional proteins [8]. For the former, the action of AMPs is believed to be initiated by cationic charges that specifically target negatively charged microbial membrane rather than mammalian cells through electrostatic interaction [9]. The difference in membrane composition between microbial and mammalian cells serves as the cornerstone for the selectivity of AMPs. The outer monolayer of microbial lipid bilayer often partially consists of phosphatidylserine (PS) or phosphatidylglycerol (PG), which provides net negative charges for microbial surfaces. In mammalian cells, however, the outer-monolayer-possessing zwitterionic phospholipids are typically not absorbed by AMPs. Subsequently, the hydrophobic portion of the peptide penetrates into the lipid bilayer, forming pores on the bacterial surface or destabilizing the plasma membrane, finally killing the bacteria [10]. The non-membranetargeting mechanism, which is capable of translocating across the membranes without damaging them but destabilizing normal cell functions [11], has received considerable attention lately, e.g., with Api137, a derivative of the insect-produced AMP apidaecin that inhibits translation by arresting the release factor on the ribosome [12]. Owing to such unique bactericidal mechanisms, it is hard for pathogens to develop resistance toward AMPs [13], rendering them promising candidates for addressing the detrimental issue of multidrug-resistant pathogens. Compared with classical antibiotics, multiple advantages are observed for AMPs - namely, (i) they are prone to synthesize since they consist usually of short amino acid sequences, which is also conducive to put into production at a relatively low cost [14]; (ii) some AMPs can bring about rapid death in Gram-positive, Gram-negative, fungi, parasites, encapsulated viruses or tumor cells merely within a few minutes [15]; (iii) they have no adverse influence on the microbiota, which is of vital significance in various aspects of human biology and is generally disrupted by conventional broadspectrum antibiotics [16]. The above-mentioned advantages make it possible to have several AMPs that are now in medical use, such as gramicidin, polymyxins, daptomycin, and melittin [17].

Nevertheless, AMPs also have drawbacks that need to be overcome. For example, AMPs show a remarkable reduction in their antimicrobial potency in the presence of biological fluids (serum and saliva), compared with non-physiological conditions (such as in phosphate buffer), which means poor bioavailability [18]. Furthermore, systemic administration results in short half-lives in vivo and cytotoxic profiles in blood [9]. Other barriers include high manufacturing cost, instability, and poorly understood pharmacokinetics, which hinder them from extensive applications [19]. Numerous previous studies have revealed the role of peptide stereochemical structure in AMPs' stability; for instance, the D-enantiomer of an oligodendrimeric M33 peptide identified from a phage-display peptide library against Escherichia coli was found to have better stability and stronger activity [20]. Based on Fan's rational design [21], in this paper, we research the incubation stability of three kinds configurations of IK8 (IK8-L, IK8-D, IK8-2D) in plasma and liver S9 of different species (human, monkey, dog, rat, and mouse), and compare the differences of metabolites of distinct configurations of IK8 in plasma and liver S9 of various species, so as to screen animal models with similar metabolic behavior to human and stable IK8 configurations in plasma and liver S9, laying a solid foundation for further in vivo experiment. 


\section{Materials and Methods}

\subsection{Materials and Chemicals}

IK8-L (IRIKIRIK- $\mathrm{NH}_{2}$-namely, all composed amino acids are in L configuration), IK8-D (IRIKIRIK-NH ${ }_{2}$-namely, all composed amino acids are in D configuration), IK8-2D (IRIkIrIK-NH $\mathrm{N}_{2}$-namely, all composed amino acids are in L configuration in addition to two which are in D configuration). The purity of these three configurations of IK8 was about $98.4 \%$ ascertained by the HPLC area normalization method, and they were kindly provided by the Zhejiang University School of Medicine. The plasma and liver S9 of five animal species (human, monkey, dog, rat, mouse) were obtained from Research Institute for Liver Diseases (Shanghai) Co. Ltd. Methanol. Acetonitrile and trifluoroacetic acid were all purchased from Aladdin Reagent Corporation (Shanghai, China).

\subsection{Sample Preparation}

\subsubsection{HPLC Sample Preparation}

IK8 was incubated in a $100 \mu \mathrm{L}$ of reaction buffer solution containing $100 \mathrm{mM}$ phosphate buffer, $1 \mathrm{U} / \mathrm{mL}$ citrate dehydrogenase, $10 \mathrm{mM}$ trisodium citrate, $10 \mathrm{mM}$ magnesium chloride, $0.5 \mathrm{mM} \mathrm{NADP}{ }^{+}$and $0.5 \mathrm{mM} \mathrm{NADPH}$, in which the volume of plasma or the content of liver S9 were $95 \mu \mathrm{L}$ or $1 \mathrm{mg} / \mathrm{mL}$, respectively. The incubation mixture was preincubated at $37^{\circ} \mathrm{C}$ for $2 \mathrm{~min}$, Three configurations of IK8 were added into plasma and liver S9 of human, monkey, dog, rat, and mouse, respectively, making the final concentration in the sample was $100 \mu \mathrm{g} / \mathrm{mL}$ and mixing with vortex evenly. The plasma and liver S9 sample of each species was prepared in duplicate. Then, incubate in $37^{\circ} \mathrm{C}$ water bath for $0 \mathrm{~min}$ and $60 \mathrm{~min}$, respectively. After that, $400 \mu \mathrm{L}$ ice acetonitrile with $2 \%$ TFA or $400 \mu \mathrm{L}$ acetonitrile with $0.5 \%$ TFA was added to the plasma system or liver S9 system, respectively, to terminate the reaction and precipitate the protein. Next, the mixture was vortexed for $1 \mathrm{~min}$ and centrifuged at 13,000 $\times g$ (Centrifuge 5424R, Eppendorf, Hamburg, Germany) at $4{ }^{\circ} \mathrm{C}$ for $15 \mathrm{~min}$. Subsequently, $30 \mu \mathrm{L}$ of supernatant was injected into the HPLC system for analysis.

\subsubsection{Q-TOF Sample Preparation}

The metabolite was collected from the above HPLC analysis effluent, and the mobile phase was evaporated to dryness under reduced pressure at $60^{\circ} \mathrm{C}$ by a centrifugal concentrator (Labconco CentriVap, Labconco Corporation, Kansas City, MO, USA). The residue was redissolved with $100 \mu \mathrm{L}$ of acetonitrile $/ \mathrm{H}_{2} \mathrm{O} /$ trifluoroacetic acid solution (50:50:0.2, $v / v / v)$ and centrifuged at $12,000 \times g$ for $10 \mathrm{~min}$ at $4{ }^{\circ} \mathrm{C}$. Finally, $5 \mu \mathrm{L}$ of supernatant was acquired for Q-TOF analysis.

\subsection{Equipment and Chromatographic Conditions}

High-performance liquid chromatography analysis was performed using an Agilent 1200 HPLC system (Agilent Technologies, Santa Clara, CA, USA) comprising a quaternary pump, a thermostatted column compartment, a solvent cabinet with degasser, an autosampler, and an ultraviolet detector. Chromatographic separation was performed on a Poroshell 120 SB-C18 $(100 \times 4.6 \mathrm{~mm}, 2.7 \mu \mathrm{m}$, Agilent Technologies, CA, USA) column at a column temperature of $35^{\circ} \mathrm{C}$ with the injection volume of $30 \mu \mathrm{L}$. The mobile phase consisted of $0.1 \%$ trifluoroacetic acid in water (A) and $0.1 \%$ trifluoroacetic acid in acetonitrile (B) at a constant flow rate of $1 \mathrm{~mL} / \mathrm{min}$ at a nonlinear gradient program as follows: The initial percentage of mobile B was 5\% remaining 3.0 min and then raised linearly to $30 \%$ in $55.0 \mathrm{~min}$, followed by a further increase to $90 \%$, maintained for $12.0 \mathrm{~min}$. The detection wavelength was $210 \mathrm{~nm}$.

Metabolite identification was performed on AB SCIEX Triple TOF 4600 mass spectrometer (AB SCIEX, Framingham, MA, USA) with an electrospray ionization source in positive ion mode. The source parameters were as follows: declustering potential, 100 $\mathrm{V}$; source temperature, $550{ }^{\circ} \mathrm{C}$; air curtain gas, $3 \mathrm{psi}$; auxiliary air pressure, $50 \mathrm{psi}$; atomization gas pressure, 50 psi. Mass spectrometer data were collected using TOF MS 
scan-information-dependent product-ion acquisition, in scan mode. The instrument was run in full scan mode (100-1200 amu). Product ion scans were performed using an m/z range of 100-1200 amu, collision energy of $40 \mathrm{eV}$, and a collision energy spread of $15 \mathrm{eV}$.

\section{Results}

\subsection{Plasma Metabolic Stability}

3.1.1. Incubation Chromatograms between Three Configurations IK8 and Plasma of Different Species (Using Rat Plasma Incubation as an Example)

By comparing the HPLC chromatogram between 0 and $60 \mathrm{~min}$ (Figure 1), it can be seen that four metabolites (M1, M2, M3, and M4) were generated after incubation of IK8-L, and only one metabolite $\left(\mathrm{M}^{\prime}\right)$ was generated for IK8-2D. In addition, there was no metabolite generated for IK8-D.

We assumed that the total peak area of metabolites M1, M2, M3, and M4 measured by HPLC was $100 \%$ so that the ratio of the peak area of each metabolite could be utilized to reflect the production of each metabolite. The principal metabolite of IK8-L in plasma was M4, followed by M2. In addition, M1 and M3 were relatively few (see Table 1).

3.1.2. Identification of Metabolites after Incubation between Three Configurations IK8 and Plasma of Different Species (Using Rat Plasma Incubation as an Example)

By comparing the HPLC chromatogram between 0 and $60 \mathrm{~min}$ (Figure 1), the possible metabolites were obvious and could be elucidated, concentrated after separation and collection, respectively. Then, the metabolites were injected into Q-TOF mass spectrometer to infer their probable structure. HPLC chromatogram of three incubated samples is shown in Figure 1. Compared with the matrix control, IK8-L showed four metabolites (Figure 1b)-M1, M2, M3, and M4. The structural information of these metabolites is presented in Figures 2-5. Likewise, according to the incubation chromatogram of IK8-2D after $60 \mathrm{~min}$ (Figure 1c), M4' was collected and injected into Q-TOF for analysis. The results demonstrated that the structure of $\mathrm{M}^{\prime}$ was also RIkIrIK-NH${ }_{2}$.

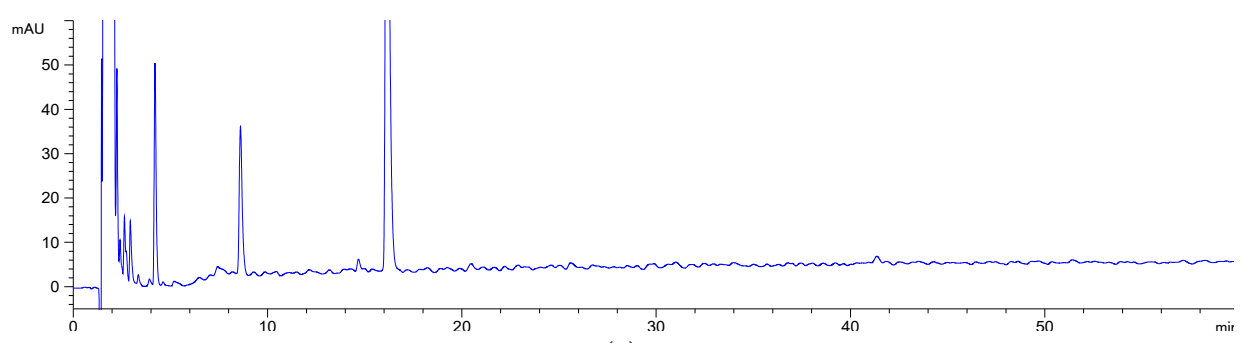

(a)

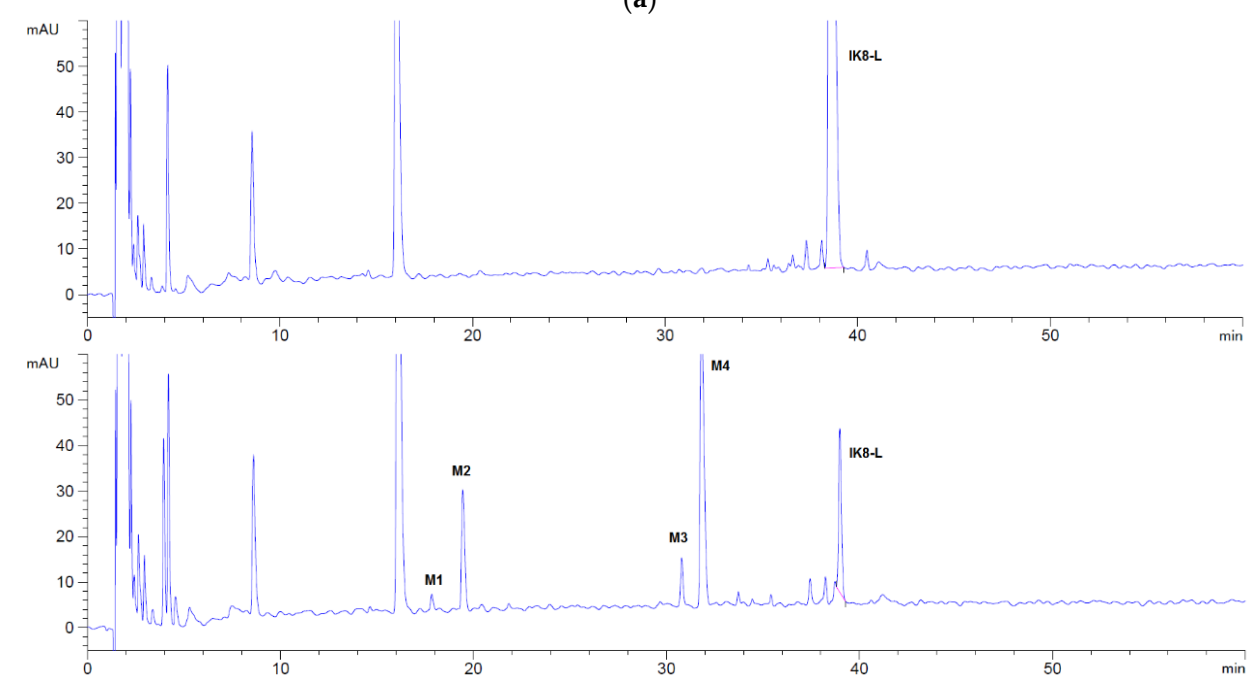

(b)

Figure 1. Cont. 


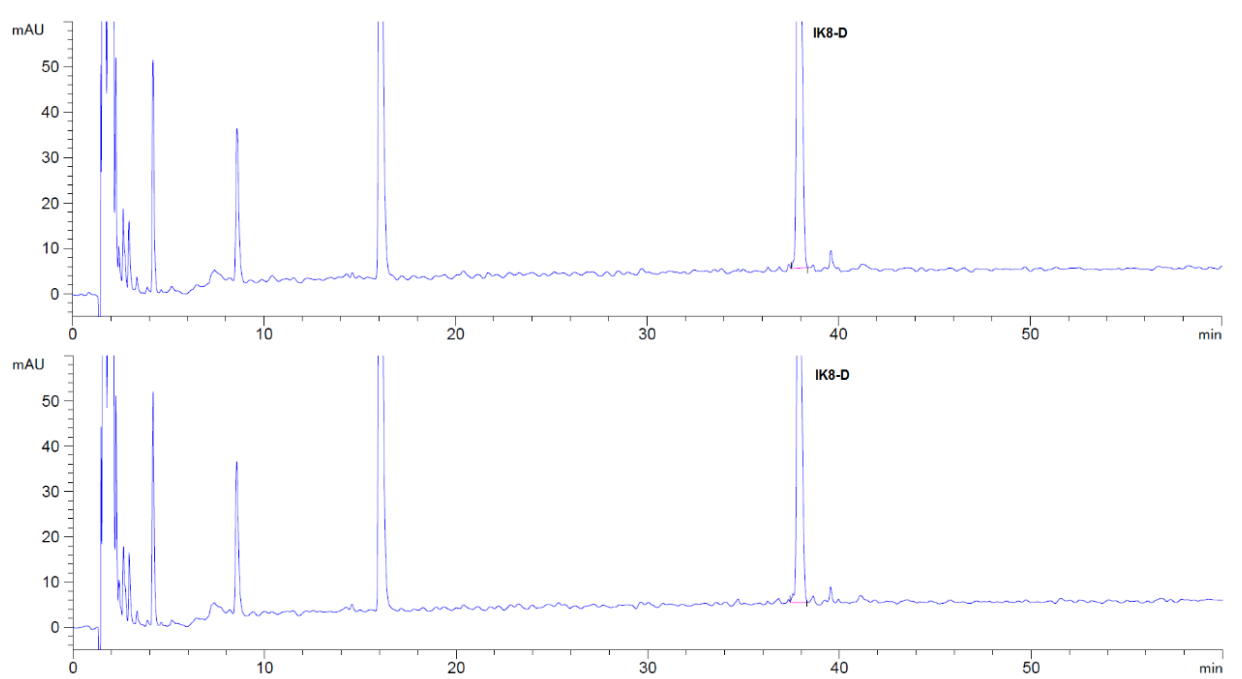

(c)

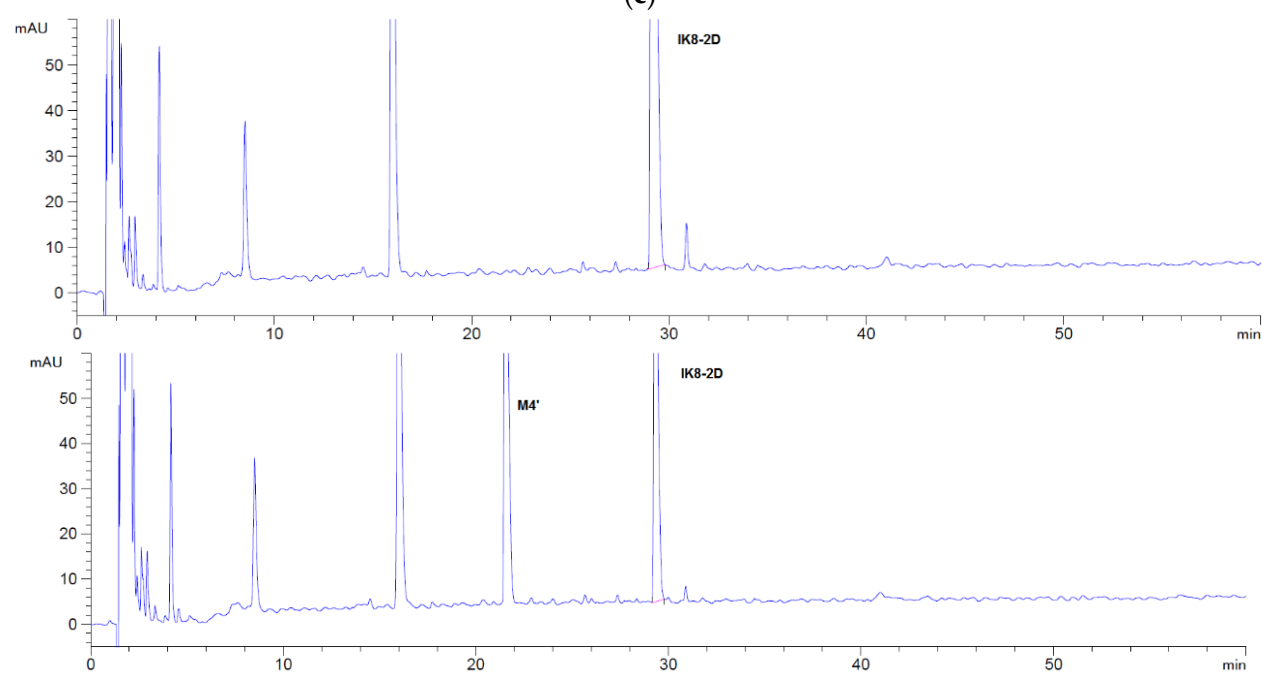

(d)

Figure 1. HPLC chromatograms of three configurations IK8 and its metabolites in plasma: (a) blank plasm; (b) IK8-L; (c) IK8-D; (d) IK8-2D.

Table 1. Relative contents of main metabolites of IK8-L in plasma of five species (\%).

\begin{tabular}{ccccc}
\hline & M1 & M2 & M3 & M4 \\
\hline Human & 2.3 & 19.1 & 11.8 & 66.8 \\
Monkey & 6.7 & 15.4 & 9.8 & 68.0 \\
Dog & ND & ND & ND & 100 \\
Rat & 2.4 & 22.2 & 6.7 & 68.7 \\
Mouse & ND & 2.5 & ND & 97.5 \\
\hline
\end{tabular}

\subsection{Liver S9 Metabolic Stability}

3.2.1. Incubation Chromatograms between Three Configurations IK8 and Liver S9 of Different Species (Using Rat Liver S9 Incubation as an Example)

By comparing the HPLC chromatogram between 0 and 60 min (Figure 6), it became apparent that night metabolites were generated after incubation of IK8-L, and two metabolites were generated for IK8-2D. In addition, there was no metabolite generated for IK8-D. As shown in Table 2, the metabolites of IK8-L incubated in liver S9 of various species had certain species distinctions. In addition to canine liver S9, M4 was the most abundant metabolite in liver S9 of the other four species. 
<smiles>CCC(C)C(N)C(=O)NC1CCCNC(=N)NC(=O)C(C(C)C)CCCC(C(N)=O)NC1=O</smiles>

\section{Exact Mass: 527.3908}

(a)

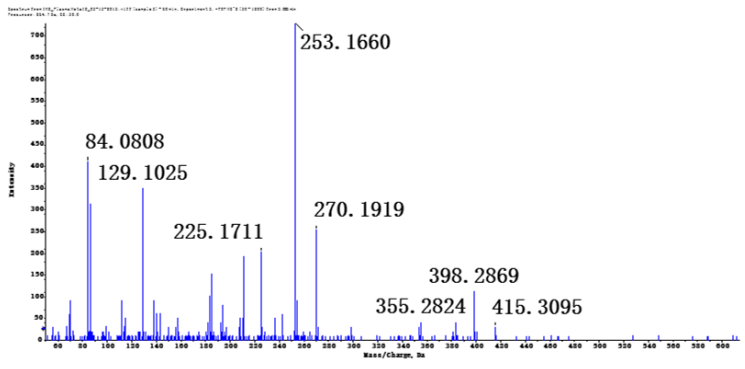

(c)

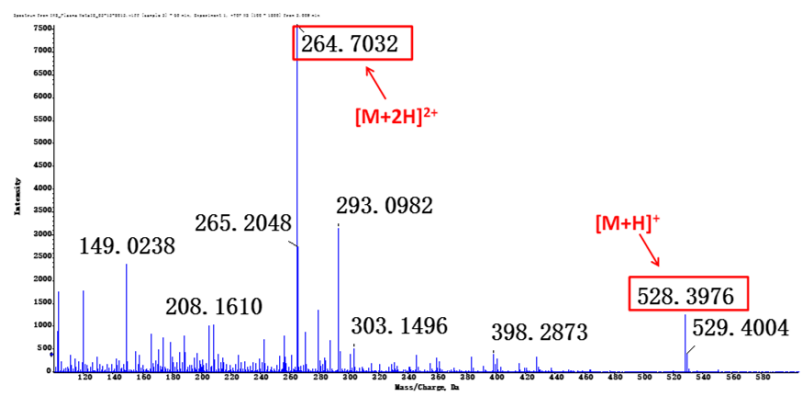

(b)

\begin{tabular}{ccccc}
\hline Name & Charge & Mass/Charge & Error (Da) & Sequence \\
\hline y1 - 17 & 1 & 129.1022 & 0.0003 & K \\
b2 - 17 & 1 & 253.1659 & 0.0000 & IR \\
b2 & 1 & 270.1925 & 0.0005 & IR \\
y3 - 17 & 1 & 398.2874 & 0.0006 & RIK \\
y3 & 1 & 415.3140 & 0.0045 & RIK \\
\hline
\end{tabular}

(d)

Figure 2. Relevant structure information of M1: (a) the structure of M1 is inferred as IRIK-NH $\mathrm{N}_{2}$ (b) primary mass spectrum of M1; (c) secondary mass spectrum of $\mathrm{M} 1\left([\mathrm{M}+2 \mathrm{H}]^{2+}\right)$; (d) the assignment of ion fragments in secondary mass spectrum.

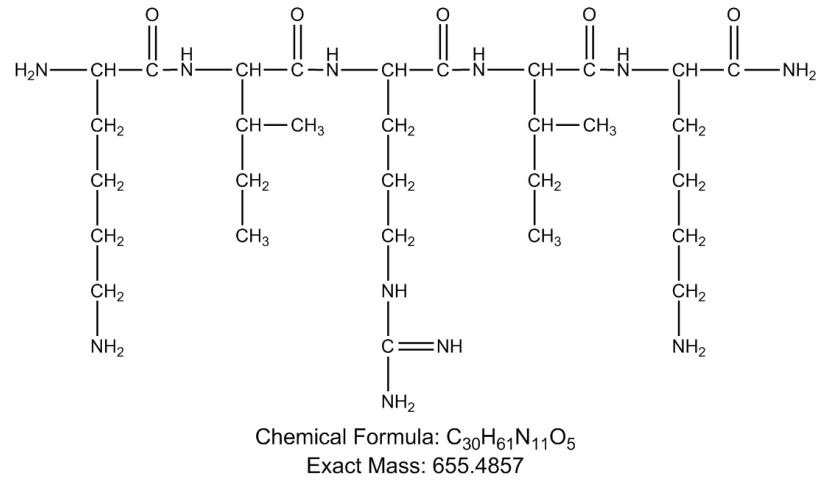

(a)

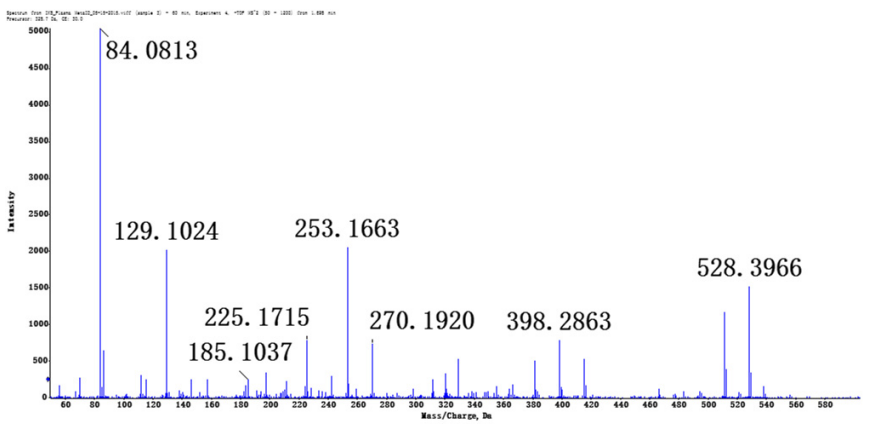

(c)

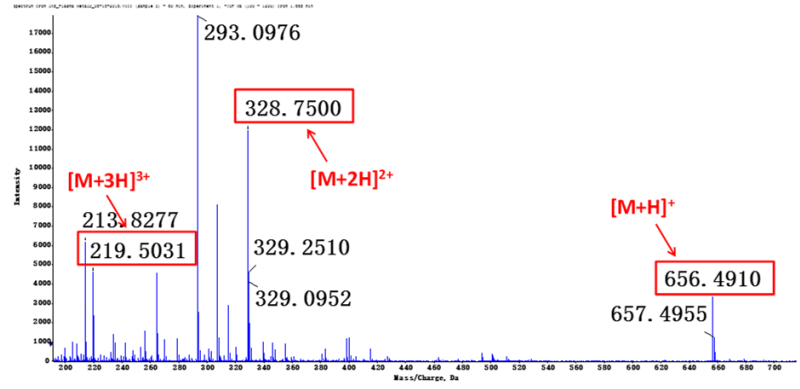

(b)

\begin{tabular}{ccccc}
\hline Name & Charge & Mass/Charge & Error (Da) & Sequence \\
\hline b1 & 1 & 129.1022 & 0.0002 & K \\
\hline y1 - 17 & 1 & 129.1022 & 0.0002 & K \\
\hline b2 - 17 & 1 & 225.1598 & 0.0117 & KI \\
\hline b3 - 17 & 1 & 381.2609 & 0.0010 & KIR \\
\hline b3 & 1 & 398.2874 & 0.0011 & KIR \\
\hline y3 - 17 & 1 & 398.2874 & 0.0011 & RIK \\
y3 & 1 & 415.3140 & 0.0010 & RIK \\
\hline y4 - 17 & 1 & 511.3715 & 0.0019 & IRIK \\
\hline b4 & 1 & 511.3715 & 0.0019 & KIRI \\
y4 & 1 & 528.3980 & 0.0014 & IRIK \\
\hline
\end{tabular}

(d)

Figure 3. Relevant structure information of M2: (a) the structure of $\mathrm{M} 2$ is inferred as $\mathrm{KIRIK}_{\mathrm{NH}}$; (b) primary mass spectrum of M2; (c) secondary mass spectrum of M2 $\left([\mathrm{M}+2 \mathrm{H}]^{2+}\right)$; (d) the assignment of ion fragments in secondary mass spectrum. 


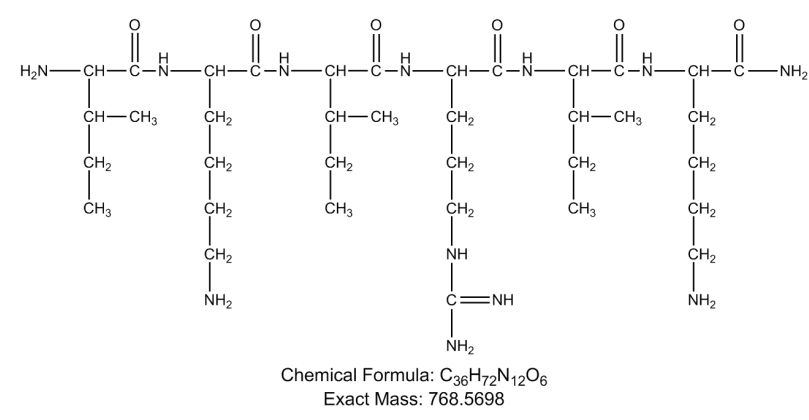

(a)

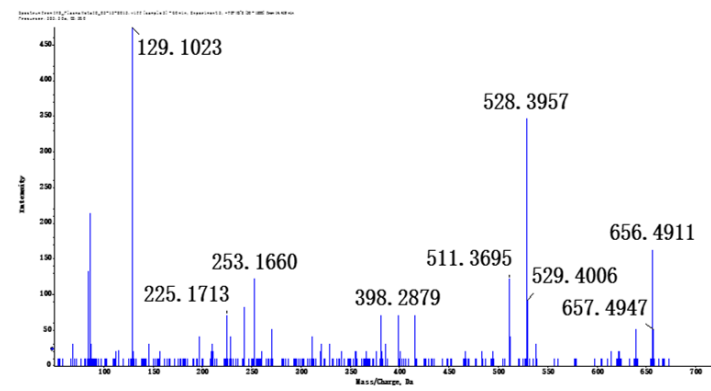

(c)

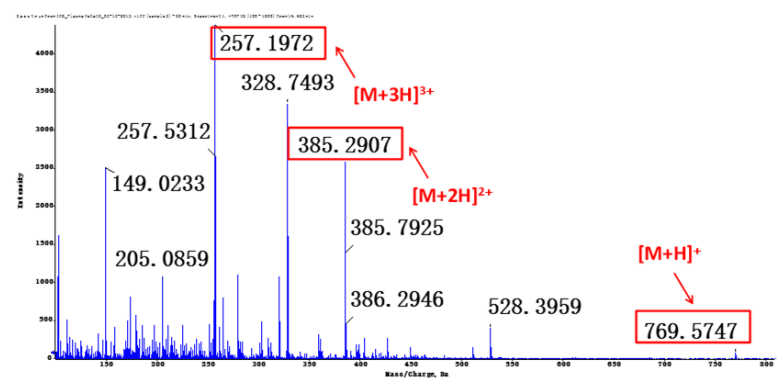

(b)

\begin{tabular}{ccccc}
\hline Name & Charge & Mass/Charge & Error (Da) & Sequence \\
\hline y1 - 17 & 1 & 129.1022 & 0.0001 & K \\
\hline b2 - 17 & 1 & 225.1598 & 0.0116 & IK \\
\hline b2 & 1 & 242.1863 & 0.0002 & IK \\
\hline y2 - 17 & 1 & 242.1863 & 0.0002 & IK \\
y3 - 17 & 1 & 398.2874 & 0.0005 & RIK \\
\hline y3 & 1 & 415.3140 & 0.0008 & RIK \\
y4 - 17 & 1 & 511.3715 & 0.0019 & IRIK \\
\hline b4 & 1 & 511.3715 & 0.0019 & IKIR \\
\hline y4 & 1 & 528.3980 & 0.0024 & IRIK \\
\hline y5 & 1 & 656.4930 & 0.0019 & KIRIK \\
\hline
\end{tabular}

(d)

Figure 4. Relevant structure information of M3: (a) the structure of M3 is inferred as IKIRIK-NH ${ }_{2}$; (b) primary mass spectrum of M3; (c) secondary mass spectrum of $\mathrm{M} 3\left([\mathrm{M}+2 \mathrm{H}]^{2+}\right)$; (d) the assignment of ion fragments in secondary mass spectrum.

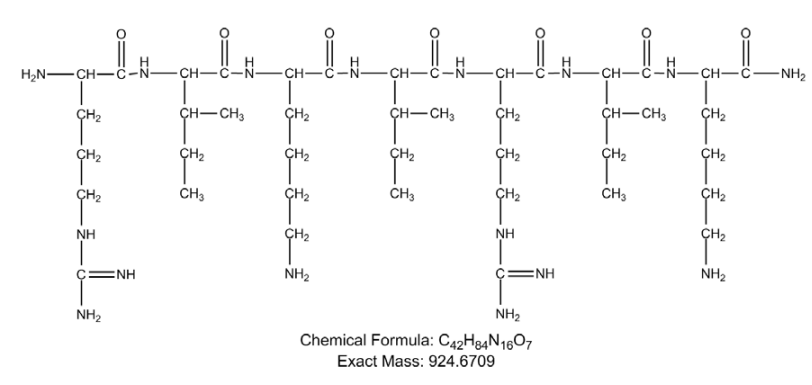

(a)

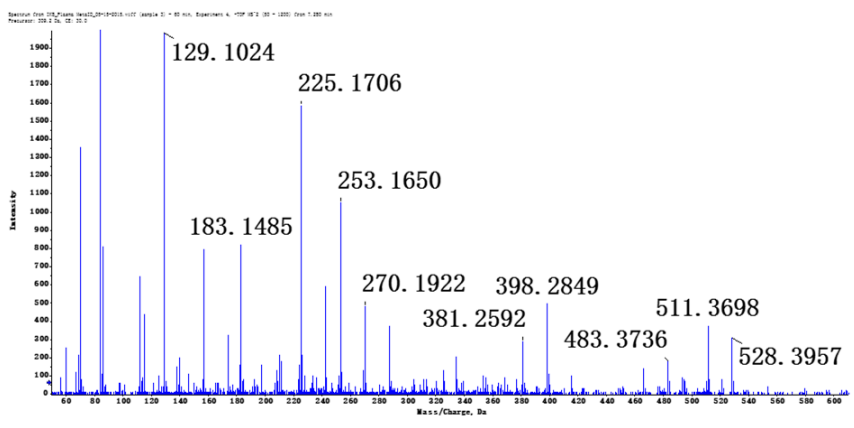

(c)

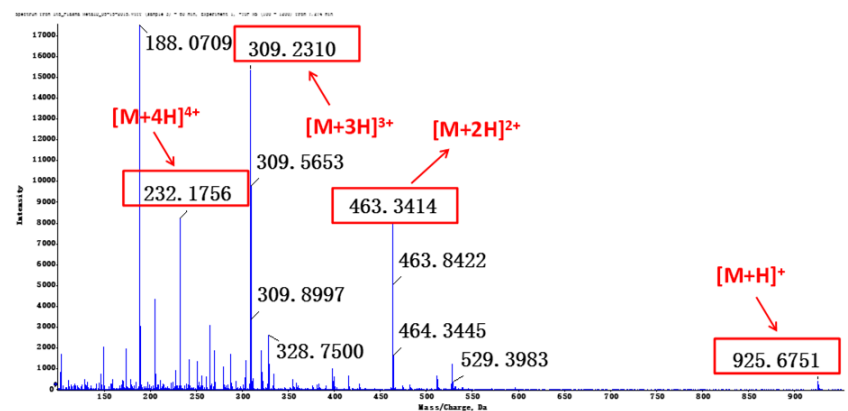

(b)

\begin{tabular}{|c|c|c|c|c|}
\hline Name & Charge & Mass/Charge & Error (Da) & Sequence \\
\hline y1 - 17 & 1 & 129.1022 & 0.0001 & K \\
\hline b1 & 1 & 157.1084 & 0.0001 & $\mathrm{R}$ \\
\hline b2 - 17 & 1 & 253.1659 & 0.0009 & RI \\
\hline b2 & 1 & 270.1925 & 0.0003 & RI \\
\hline b3 - 17 & 1 & 381.2609 & 0.0016 & RIK \\
\hline b3 & 1 & 398.2874 & 0.0025 & RIK \\
\hline y3 - 17 & 1 & 398.2874 & 0.0025 & RIK \\
\hline b4 & 1 & 511.3715 & 0.0017 & RIKI \\
\hline$y 4-17$ & 1 & 511.3715 & 0.0017 & IRIK \\
\hline $\mathrm{y} 4$ & 1 & 528.3980 & 0.0023 & IRIK \\
\hline
\end{tabular}

(d)

Figure 5. Relevant structure information of M4: (a) the structure of M4 is inferred as RIKIRIK-NH 2 ; (b) primary mass spectrum of M4; (c) secondary mass spectrum of M4 $\left([\mathrm{M}+2 \mathrm{H}]^{2+}\right)$; (d) the assignment of ion fragments in secondary mass spectrum. 


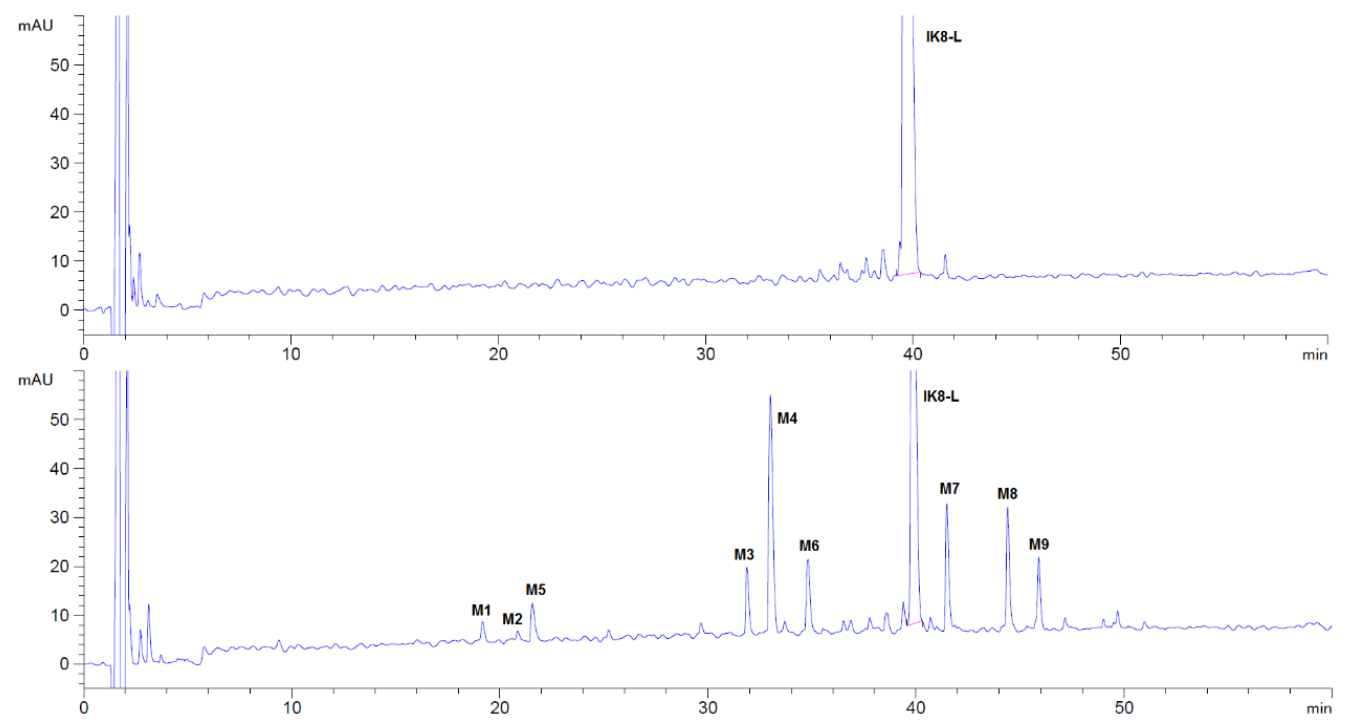

(a)
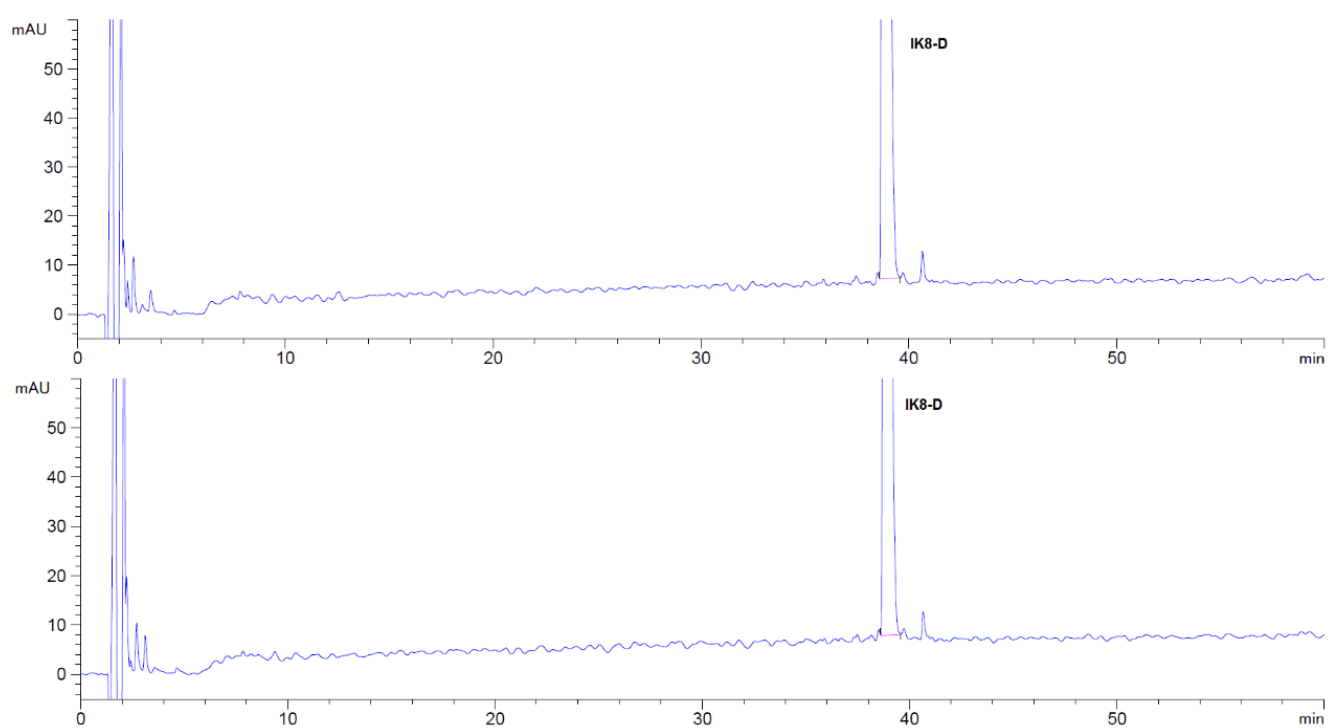

(b)
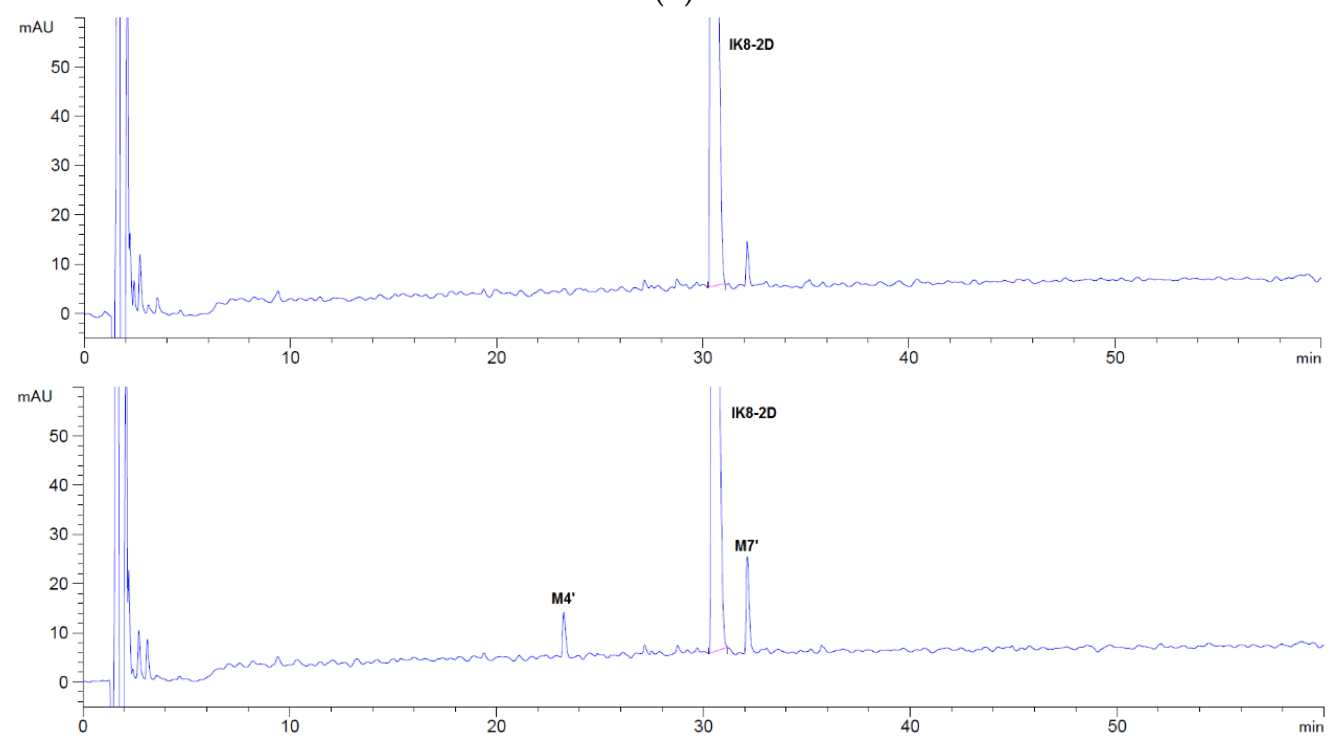

(c)

Figure 6. HPLC chromatograms of three configurations IK8 and its metabolites in liver S9: (a) IK8-L; (b) IK8-D; (c) IK8-2D. 
Table 2. Relative contents of main metabolites of IK8-L in liver S9 of five species (\%).

\begin{tabular}{|c|c|c|c|c|c|c|c|c|c|}
\hline & M1 & M2 & M3 & M4 & M5 & M6 & M7 & M8 & M9 \\
\hline Human & 4.0 & 3.9 & 5.3 & 62.0 & 1.8 & 7.5 & 4.8 & 4.5 & 6.3 \\
\hline Monkey & 2.5 & 0.0 & 2.2 & 44.1 & 4.6 & 18.9 & 9.0 & 6.5 & 12.2 \\
\hline Dog & 9.2 & 0.0 & 4.9 & 17.5 & 23.2 & 11.2 & 14.0 & 12.0 & 8.0 \\
\hline Rat & 2.1 & 1.0 & 7.7 & 35.2 & 5.8 & 9.5 & 14.7 & 14.2 & 9.9 \\
\hline Mouse & 4.5 & 0.0 & 3.7 & 67.6 & 3.7 & 4.8 & 7.4 & 5.5 & 2.8 \\
\hline
\end{tabular}

3.2.2. Identification of Metabolites after Incubation between Three Configurations IK8 and Liver S9 of Different Species (Using Rat Liver S9 Incubation as an Example)

The HPLC chromatograms of IK8-L, IK8-D, and IK8-2D incubated with rat liver S9 are shown in Figure 6. Compared with the results in rat plasma (Figure 1), there were five more metabolites of IK8-L in rat liver S9. The results indicated that the metabolites M1, M2, M3, and M4 were identical to that in plasma. Moreover, the structural information of the rest metabolites-M5, M6, and M7-are presented in Figures 7-9. Unfortunately, the structures of M8 and M9 were not identified by the Q-TOF data. For IK8-2D, it can be illustrated, through primary and secondary mass spectrometry, that the structures of M4' and $\mathrm{M}^{\prime}$ ' were RIkIrIK-NH${ }_{2}$ and IRIkIrIK, respectively.

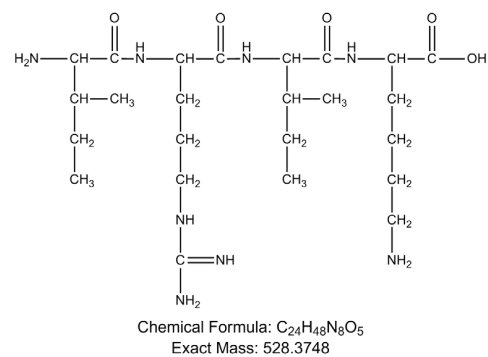

(a)

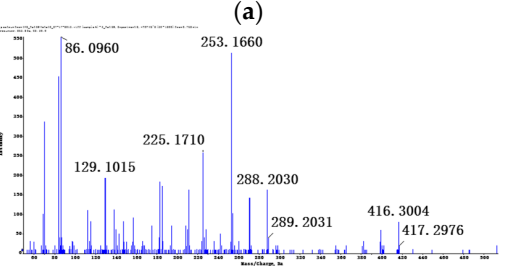

(c)

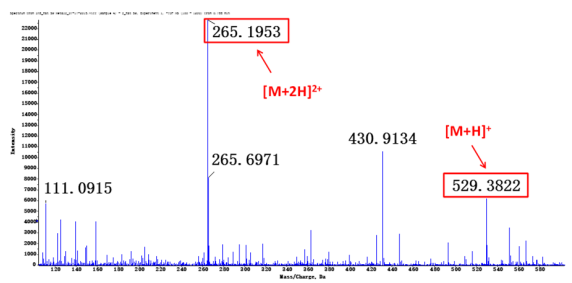

(b)

\begin{tabular}{ccccc}
\hline Name & Charge & Mass/Charge & Error (Da) & Sequence \\
\hline y1 - 18 & 1 & 129.1022 & 0.0007 & $\mathrm{~K}$ \\
y1 - 17 & 1 & 130.0863 & 0.0007 & $\mathrm{~K}$ \\
b2 - 17 & 1 & 253.1659 & 0.0001 & IR \\
y3 - 17 & 1 & 399.2714 & 0.0007 & RIK \\
y3 & 1 & 416.2980 & 0.0024 & RIK \\
\hline
\end{tabular}

(d)

Figure 7. Relevant structure information of M5: (a) the structure of M5 is inferred as IRIK; (b) primary mass spectrum of M5; (c) secondary mass spectrum of M5 $\left([\mathrm{M}+2 \mathrm{H}]^{2+}\right)$; $(\mathbf{d})$ the assignment of ion fragments in secondary mass spectrum.

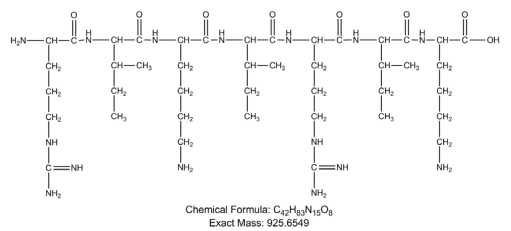

(a)

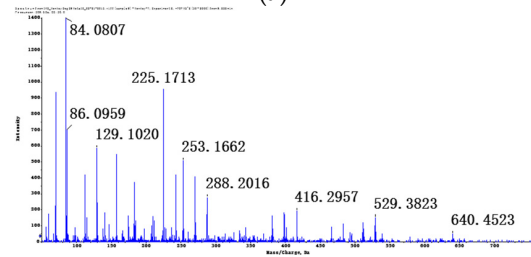

(c)

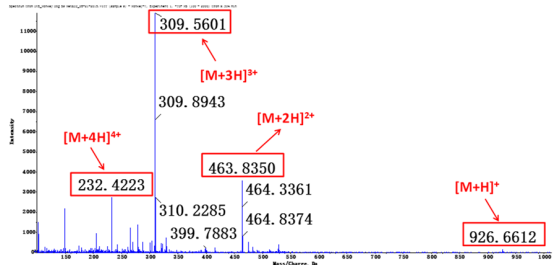

(b)

\begin{tabular}{ccccc}
\hline Name & Charge & Mass/Charge & Error (Da) & Sequence \\
\hline y1 - 18 & 1 & 129.1022 & 0.0003 & $\mathrm{~K}$ \\
\hline $\mathrm{b} 2-17$ & 1 & 253.1659 & 0.0003 & $\mathrm{RI}$ \\
$\mathrm{y} 3$ & 1 & 416.2980 & 0.0023 & RIK \\
y4 & 1 & 529.3820 & 0.0003 & IRIK \\
y5 - 17 & 1 & 640.4505 & 0.0018 & KIRIK \\
\hline
\end{tabular}

(d)

Figure 8. Relevant structure information of M6: (a) the structure of M6 is inferred as RIKIRIK; (b) primary mass spectrum of M6; (c) secondary mass spectrum of $\mathrm{M6}\left([\mathrm{M}+3 \mathrm{H}]^{3+}\right)$; (d) the assignment of ion fragments in secondary mass spectrum. 


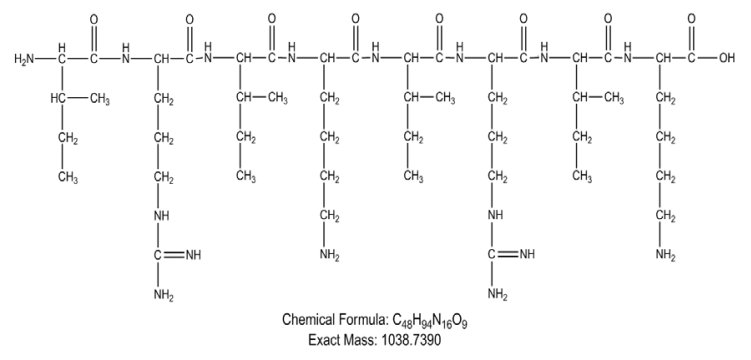

(a)

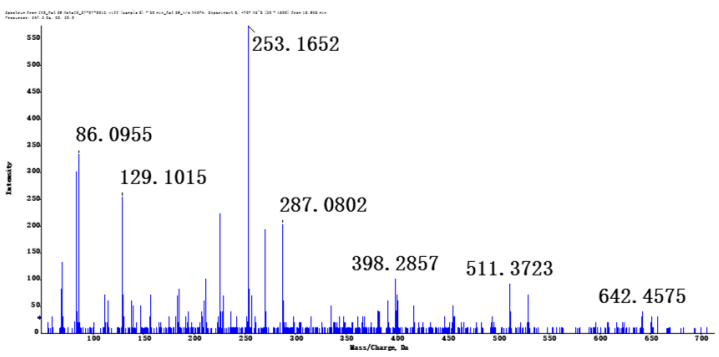

(c)

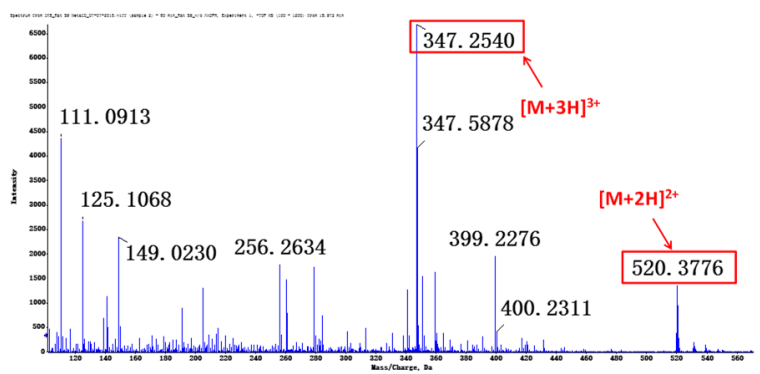

(b)

\begin{tabular}{ccccc}
\hline Name & Charge & Mass/Charge & Error (Da) & Sequence \\
\hline y1 - 18 & 1 & 129.1022 & 0.0007 & K \\
\hline b2 - 17 & 1 & 253.1659 & 0.0007 & IR \\
\hline b2 & 1 & 270.1925 & 0.0014 & IR \\
\hline y3 - 18 & 1 & 398.2874 & 0.0017 & RIK \\
\hline b4 & 1 & 511.3715 & 0.0009 & IRIK \\
\hline y4 - 18 & 1 & 511.3715 & 0.0009 & IRIK \\
y8 - 18 & 2 & 511.3715 & 0.0009 & IRIKIRIK \\
\hline b8 & 2 & 511.3715 & 0.0009 & IRIKIRIK \\
\hline y4 & 1 & 529.3820 & 0.0028 & IRIK \\
\hline
\end{tabular}

(d)

Figure 9. Relevant structure information of M7: (a) the structure of M7 is inferred as IRIKIRIK; (b) primary mass spectrum of M7 $\left([\mathrm{M}+2 \mathrm{H}]^{2+}\right)$; (c) secondary mass spectrum of M7 $\left([\mathrm{M}+3 \mathrm{H}]^{3+}\right)$; $(\mathbf{d})$ the assignment of ion fragments in secondary mass spectrum.

\section{Discussion}

One of the major factors confining the clinical utility of AMPs resides in their instability, especially their susceptibility to the degradation by proteases [22]. In this paper, we aimed to elucidate the relationship between configurations of IK8 and its stability through analyzing metabolites. The results suggested that the stability of three configurations of IK8 in five species of plasma was IK8-L < IK8-2D $<$ IK8-D, which was the same as that in liver S9. On the one hand, as incubating in the plasma of various species, four metabolites were produced for IK8-L, among which the maximum metabolite was M4 (RIKIRIK-NH $H_{2}$ ); one metabolite was produced for IK8-2D, which was also M4 (RIKIRIK$\mathrm{NH}_{2}$ ). On the other hand, as for incubation in liver $\mathrm{S} 9$ of various species, nine metabolites were produced for IK8-L. Apart from dog liver S9, M4 (RIKIRIK-NH ${ }_{2}$ ) was the most abundant metabolite in liver S9 of the other four species; two metabolites were produced for IK8-2D. Further, a shared phenomenon was revealed that regardless of whether in plasma or liver S9, no metabolites of IK8-D were detected. Incidentally, our major work in this paper focused on identifying the metabolites with retention time after 16 min; therefore, the possible metabolites before this time remain to be determined. Interestingly, only peptide bond hydrolytic metabolites of IK8-L were found in plasma incubation solution (M1, M2, M3, and M4). However, in liver S9 incubation solution, except for peptide bond hydrolysis metabolites, metabolites with terminal amide bonds hydrolyzed to carboxyl groups were also found (M5, M6, and M7), which was employed to remove the carboxyterminal negative charge and particularly render a high net positive charge for enhanced antimicrobial activities.

In view of these results, we believed that the introduction of D-amino acid plays a crucial role in promoting the stability of the polypeptide, which is consistent with Chiara's findings [20]. Importantly, according to our published report [21], the substitution of peptide IK8-L with D-amino acids considerably strengthened antimicrobial activities. Moreover, among the three configuration peptides referred in this study, IK8-D demonstrated the most potent antimicrobial activities against five types of microorganisms (S. epidermidis, S. aureus, E. coli, P. aeruginosa, and C. albicans), with the lowest geometric mean of minimum inhibitory concentrations (MIC) value of $4.3 \mathrm{mg} / \mathrm{L}$, while the values of IK8-L and IK8-2D under identical testing conditions were $23.4 \mathrm{mg} / \mathrm{L}$ and $12.5 \mathrm{mg} / \mathrm{L}$, respectively. 
In terms of toxicity study, several years ago, we initially used $4 \%$ (by volume) rabbit blood to evaluate the hemolytic activities of these three IK8 forms [21], and the results suggested that they all exhibited minimal hemolysis against the highly sensitive erythrocytes at their corresponding MIC values, indicating prominent hemocompatibility. Meanwhile, only slight differences existed in hemolytic activities among three enantiomeric forms of IK8, with the $\mathrm{HC}_{10}$ values (defined as the lowest peptide concentration that induced $10 \%$ or more hemolysis) of IK8-L, IK8-2D and IK8-D observed to be 2000, 1600, and $1750 \mathrm{mg} / \mathrm{L}$, respectively. To further verify the safety, the cytotoxic test of three IK8 configurations on human keratinocytes (HaCat cells) was subsequently carried out via the MTT assay [23], and the obtained statistic revealed that viability percentages of human HaCaT cells after incubation with these three IK8 forms were all more than $90 \%$, up to $500 \mathrm{mg} / \mathrm{L}$, which significantly exceeded the maximal MIC of three IK8 forms toward numerous bacteria such as S. epidermidis and E. coli. Therefore, on the basis of enhanced antimicrobial activities and low toxicity, which we have proved previously, along with the superior stability we presented in this paper, convincing evidence revealed that D-amino acids substituting peptide IK8-D is a promising therapeutic candidate with the potential to combat antibiotics resistant microorganisms and ultimately improve the curative effect.

Author Contributions: Conceptualization, L.Y. and N.L.; methodology, Y.M. and S.Z. (Su Zeng); formal analysis, Y.M. and M.X.; investigation, Y.M., S.Z. (Shaojun Zhou), and M.X.; resources, W.F.; data curation, M.X.; writing-original draft preparation, S.Z. (Shaojun Zhou); writing-review and editing, L.Y. All authors have read and agreed to the published version of the manuscript.

Funding: This study was supported by the Key Research and Development Program of Zhejiang Province, China (2020C03045), and the Leading Talent of "Ten Thousand Plan"-National High-Level Talents Special Support Plan.

Institutional Review Board Statement: Not applicable.

Informed Consent Statement: Not applicable.

Data Availability Statement: Not applicable.

Conflicts of Interest: The authors declare no conflict of interest.

\section{References}

1. Lei, J.; Sun, L.; Huang, S.; Zhu, C.; Li, P.; He, J.; Mackey, V.; Coy, D.H.; He, Q. The antimicrobial peptides and their potential clinical applications. Am. J. Transl. Res. 2019, 11, 3919-3931. [PubMed]

2. Friedman, N.D.; Temkin, E.; Carmeli, Y. The negative impact of antibiotic resistance. Clin. Microbiol. Infect. Off. Publ. Eur. Soc. Clin. Microbiol. Infect. Dis. 2016, 22, 416-422. [CrossRef] [PubMed]

3. Rima, M.; Rima, M.; Fajloun, Z.; Sabatier, J.M.; Bechinger, B.; Naas, T. Antimicrobial peptides: A potent alternative to antibiotics. Antibiotics 2021, 10, 1095. [CrossRef]

4. Essig, A.; Hofmann, D.; Münch, D.; Gayathri, S.; Künzler, M.; Kallio, P.T.; Sahl, H.G.; Wider, G.; Schneider, T.; Aebi, M. Copsin, a novel peptide-based fungal antibiotic interfering with the peptidoglycan synthesis. J. Biol. Chem. 2014, 289 , 34953-34964. [CrossRef] [PubMed]

5. Wu, Q.; Patočka, J.; Kuča, K. Insect antimicrobial peptides, a mini review. Toxins 2018, 10, 461. [CrossRef] [PubMed]

6. Wang, S.; Fang, Q.; Lu, Z.; Gao, Y.; Trembleau, L.; Ebel, R.; Andersen, J.H.; Philips, C.; Law, S.; Deng, H. Discovery and biosynthetic investigation of a new antibacterial dehydrated non-ribosomal tripeptide. Angew. Chem. 2021, 60, 3229-3237. [CrossRef]

7. Magana, M.; Pushpanathan, M.; Santos, A.L.; Leanse, L.; Fernandez, M.; Ioannidis, A.; Giulianotti, M.A.; Apidianakis, Y.; Bradfute, S.; Ferguson, A.L.; et al. The value of antimicrobial peptides in the age of resistance. Lancet. Infect. Dis. 2020, 20, e216-e230. [CrossRef]

8. Erdem Büyükkiraz, M.; Kesmen, Z. Antimicrobial peptides (AMPs): A promising class of antimicrobial compounds. J. Appl. Microbiol. 2021. Online ahead of print. [CrossRef] [PubMed]

9. Kumar, P.; Kizhakkedathu, J.N.; Straus, S.K. Antimicrobial peptides: Diversity, mechanism of action and strategies to improve the activity and biocompatibility in vivo. Biomolecules 2018, 8, 4. [CrossRef] [PubMed]

10. Cheng, J.; Chin, W.; Dong, H.; Xu, L.; Zhong, G.; Huang, Y.; Li, L.; Xu, K.; Wu, M.; Hedrick, J.L.; et al. Biodegradable antimicrobial polycarbonates with in vivo efficacy against multidrug-resistant MRSA systemic infection. Adv. Healthc. Mater. 2015, 4, $2128-2136$. [CrossRef] [PubMed]

11. Boparai, J.K.; Sharma, P.K. Mini review on antimicrobial peptides, sources, mechanism and recent applications. Protein Pept. Lett. 2020, 27, 4-16. [CrossRef] [PubMed] 
12. Florin, T.; Maracci, C.; Graf, M.; Karki, P.; Klepacki, D.; Berninghausen, O.; Beckmann, R.; Vázquez-Laslop, N.; Wilson, D.N.; Rodnina, M.V.; et al. An antimicrobial peptide that inhibits translation by trapping release factors on the ribosome. Nat. Struct. Mol. Biol. 2017, 24, 752-757. [CrossRef] [PubMed]

13. Zhang, Q.Y.; Yan, Z.B.; Meng, Y.M.; Hong, X.Y.; Shao, G.; Ma, J.J.; Cheng, X.R.; Liu, J.; Kang, J.; Fu, C.Y. Antimicrobial peptides: Mechanism of action, activity and clinical potential. Mil. Med. Res. 2021, 8, 48. [CrossRef] [PubMed]

14. Hansen, I.; Lövdahl, T.; Simonovic, D.; Hansen, K.; Andersen, A.J.C.; Devold, H.; Richard, C.S.M.; Andersen, J.H.; Strøm, M.B.; Haug, T. Antimicrobial activity of small synthetic peptides based on the marine peptide turgencin A: Prediction of antimicrobial peptide sequences in a natural peptide and strategy for optimization of potency. Int. J. Mol. Sci. 2020, 21, 5460. [CrossRef]

15. Raheem, N.; Straus, S.K. Mechanisms of action for antimicrobial peptides with antibacterial and antibiofilm functions. Front. Microbiol. 2019, 10, 2866. [CrossRef]

16. Kim, H.; Jang, J.H.; Kim, S.C.; Cho, J.H. Development of a novel hybrid antimicrobial peptide for targeted killing of Pseudomonas aeruginosa. Eur. J. Med. Chem. 2020, 185, 111814. [CrossRef]

17. Dijksteel, G.S.; Ulrich, M.M.W.; Middelkoop, E.; Boekema, B. Review: Lessons learned from clinical trials using antimicrobial peptides (AMPs). Front. Microbiol. 2021, 12, 616979. [CrossRef] [PubMed]

18. Mahlapuu, M.; Håkansson, J.; Ringstad, L.; Björn, C. Antimicrobial peptides: An emerging category of therapeutic agents. Front. Cell. Infect. Microbiol. 2016, 6, 194. [CrossRef]

19. Chin, W.; Zhong, G.; Pu, Q.; Yang, C.; Lou, W.; De Sessions, P.F.; Periaswamy, B.; Lee, A.; Liang, Z.C.; Ding, X.; et al. A macromolecular approach to eradicate multidrug resistant bacterial infections while mitigating drug resistance onset. Nat. Commun. 2018, 9, 917. [CrossRef] [PubMed]

20. Falciani, C.; Lozzi, L.; Pollini, S.; Luca, V.; Carnicelli, V.; Brunetti, J.; Lelli, B.; Bindi, S.; Scali, S.; Di Giulio, A.; et al. Isomerization of an antimicrobial peptide broadens antimicrobial spectrum to gram-positive bacterial pathogens. PLoS ONE 2012, 7, e46259. [CrossRef] [PubMed]

21. Ong, Z.Y.; Cheng, J.; Huang, Y.; Xu, K.; Ji, Z.; Fan, W.; Yang, Y.Y. Effect of stereochemistry, chain length and sequence pattern on antimicrobial properties of short synthetic $\beta$-sheet forming peptide amphiphiles. Biomaterials 2014, 35, 1315-1325. [CrossRef] [PubMed]

22. Greco, I.; Molchanova, N.; Holmedal, E.; Jenssen, H.; Hummel, B.D.; Watts, J.L.; Håkansson, J.; Hansen, P.R.; Svenson, J. Correlation between hemolytic activity, cytotoxicity and systemic in vivo toxicity of synthetic antimicrobial peptides. Sci. Rep. 2020, 10, 13206. [CrossRef] [PubMed]

23. Zhong, G.; Cheng, J.; Liang, Z.C.; Xu, L.; Lou, W.; Bao, C.; Ong, Z.Y.; Dong, H.; Yang, Y.Y.; Fan, W. Short Synthetic $\beta$-Sheet Antimicrobial Peptides for the Treatment of Multidrug-Resistant Pseudomonas aeruginosa Burn Wound Infections. Adv. Healthc. Mater. 2017, 6, 1601134. [CrossRef] [PubMed] 